\title{
2', 3-dihydroxy-5-methoxybiphenyl suppresses fMLP-induced superoxide anion production and cathepsin $G$ release by targeting the beta-subunit of G-protein in human neutrophils
}

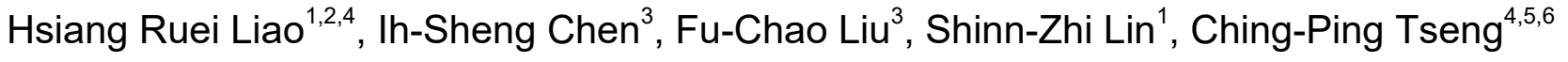 \\ ${ }^{I}$ Graduate Institute of Natural Products, Chang-Gunag University, Taiwan, ${ }^{2}$ Department of Anesthesiology, Chang \\ Gung Memorial Hospital, Taiwan, ${ }^{3}$ Graduate Institute of Natural Products, College of Pharmacy, Kaohsiung Medical \\ University, Taiwan, ${ }^{4}$ Graduate Institute of Biomedical Sciences, College of Medicine, Chang Gung University, Taiwan, \\ ${ }^{5}$ Department of Medical Biotechnology and Laboratory Science, College of Medicine, Chang Gung University, Taiwan, \\ ${ }^{6}$ Department of Laboratory Medicine, Chang Gung Memorial Hospital, Taiwan
}

This study investigates the effect and the underlying mechanism of 2',3-dihydroxy-5-methoxybiphenyl (RIR-2), a lignan extracted from the roots of Rhaphiolepis indica (L.) Lindl. ex Ker var. tashiroi Hayata ex Matsum. \& Hayata (Rosaceae), on N-formyl-L-methionyl-L-leucyl-L-phenylalanine (fMLP)-induced respiratory burst and cathepsin $\mathrm{G}$ in human neutrophils. However, 2',3-dihydroxy-5-methoxybiphenyl (RIR-2) was not found to inhibit PMA-induced respiratory bust and cathepsin G release. Signaling pathways regulated by RIR2 which modulated fMLP-induced respiratory burst and cathepsin $\mathrm{G}$ release were evaluated by an interaction between $\mathrm{G} \beta$-protein with downstream signaling induced by fMLP and by immunoblotting analysis of the downstream targets of $\mathrm{G} \beta$-protein. Briefly, 2',3-dihydroxy-5-methoxybiphenyl (RIR-2) inhibited fMLP-induced superoxide anion production (IC50=2.57 $\pm 0.22 \mu \mathrm{M}, \mathrm{n}=4$ ), cathepsin $\mathrm{G}$ release (IC50=18.72 $\pm 3.76 \mu \mathrm{M}, \mathrm{n}=4$ ) and migration in a concentration dependent manner. Further, 2',3-dihydroxy-5methoxybiphenyl (RIR-2) specifically suppresses fMLP-induced Src family kinases phosphorylation, -Raf/ERK phosphorylation, by inhibiting an interaction between $\mathrm{G} \beta$-protein with Src kinases without inhibiting Src kinases activities. Consequently, 2',3-dihydroxy-5-methoxybiphenyl (RIR2) attenuated the downstream targets of Src kinase, such as Tec translocation from the cytosol to the inner leaflet of the plasma membrane, phosphorylation of AKT, P38, PLC $\gamma 2$, PKC and membrane localization of p47phox and P40phox, furthermore, 2',3-dihydroxy-5-methoxybiphenyl (RIR-2) attenuated fMLP-induced intracellular calcium mobilization, moreover, 2',3-dihydroxy-5-methoxybiphenyl (RIR-2) was not found to increase cyclicAMP. 2',3-dihydroxy-5-methoxybiphenyl (RIR2) was not a competitive or allosteric antagonist of fMLP. On the other hand, PMA-induced phosphorylation of Src, AKT, P38, PKC and membrane localization of p47phox and P40phox remained unaffected. In conclusion, 2',3-dihydroxy-5-methoxybiphenyl (RIR2) specifically modulates fMLP-mediated neutrophil superoxide anion production and cathepsin $G$ release by inhibiting an interaction between $\mathrm{G} \beta$-protein with downstream signaling which subsequently interferes with the activation of intracellular calcium, PLC $\gamma 2$, AKT, p38, PKC, ERK, p47phox and p40phox. 